Gut, 1984, 25, 1057-1064

\title{
Impaired oxidation of debrisoquine in patients with perhexiline liver injury
}

\author{
MARSHA Y MORGAN, R RESHEF, R R SHAH, N S OATES, \\ R L SMITH, AND SHEILA SHERLOCK \\ From the Department of Medicine, The Royal Free Hospital School of Medicine, London, and \\ Department of Pharmacology, St Mary's Hospital Medical School, London
}

SUMmaRY Perhexiline maleate is an antianginal agent which depends on hepatic oxidation for its elimination. Its use may be complicated by the development of peripheral neuropathy and liver damage. The majority of patients with perhexiline neuropathy have an impaired ability to effect metabolic drug oxidation which is genetically determined. Information has not been available on drug oxidation capacity in patients with perhexiline liver injury. Drug oxidation was measured using an oxidation phenotyping procedure in four patients with perhexiline liver injury and in 70 patients with chronic liver disease serving as a control group. All four patients with perhexiline liver damage showed a substantial metabolic defect; three of the four patients $(75 \%)$ showed a genetically determined impairment of oxidation capacity. The incidence of severely impaired oxidation capacity in the perhexiline group was significantly greater than in the patients with chronic liver disease $(6 / 70 ; 8.6 \%)$ and in the healthy population $(9 \%)(F=0.0048)$. A clear association exists between perhexiline liver injury and diminished drug metabolic activity, suggesting that the propensity to develop perhexiline liver injury is, at least in part, genetically determined.

Perhexiline maleate (Pexid) has been used for the treatment of angina pectoris since 1974. Although clinical trials have shown its undoubted efficacy, ${ }^{-3}$ its use is associated with serious side effects such as weight loss,${ }^{45}$ hypoglycaemia,${ }^{6}$ proximal myopathy, ${ }^{7}$ peripheral neuropathy ${ }^{89}$ and hepatic damage. ${ }^{10-12}$ The neuropathy and the hepatic damage may not reverse when the drug is withdrawn and deaths have been recorded. ${ }^{11-18}$

Severe adverse reactions follow the use of perhexiline in only a percentage of patients and are perhaps associated with impaired metabolism of the drug. Perhexiline is a lipophilic drug and its elimination depends on hepatic oxidation to the more polar monohydroxylated and dihydroxylated metabolites. ${ }^{19}$ Several drug oxidations in man are under single gene control and exhibit genetic polymorphism. ${ }^{20} 21$ Oxidation is regulated by two alleles, $\mathrm{D}^{\mathrm{H}}$-rapid and extensive oxidation and $\mathrm{D}^{\mathrm{L}}$. slow impaired oxidation. ${ }^{21}$ Individuals homozygous for the $\mathrm{D}^{\mathrm{L}}$ allele have an impaired ability to.oxidise many drugs. Studies indicate that $9 \%$ of the British

Address for correspondence: Dr M Y Morgan. Department of Medicine, Royal Free Hospital, Hampstead, London NW3 2QG.

Received for publication 5 December 1983 white population have a genetically determined inability to effect metabolic drug oxidation. ${ }^{21}$ For technical reasons drug oxidising ability cannot be measured using perhexiline but is readily determined using debrisoquine. ${ }^{21} 22$ It has been shown recently that the majority of patients who develop perhexiline neuropathy show impaired drug oxidising ability suggesting that the susceptibility to perhexiline neuropathy is largely genetically determined. ${ }^{23}$ No study of a similar nature has been undertaken in patients with perhexiline related liver injury.

This paper reports the results of oxidation phenotyping in four patients with perhexiline liver injury. Liver damage itself may be associated with impaired oxidation capacity ${ }^{24}$ thus 70 patients with chronic liver disease were studied as a control group. Patients with alcohol related liver injury were excluded from the control group because an individual's susceptibility to develop alcohol related liver injury is probably at least in part genetically determined. Although ethanol is metabolised mainly by the alcohol dehydrogenase pathway there is evidence to suggest that at high ethanol concentrations the $\mathrm{P} 450$ system contributes. ${ }^{25}$ It is conceivable that a genetic impairment of oxidation 
capacity might predispose to alcohol related liver injury.

\section{Case histories}

TB, a man aged 54 years, was admitted in March 1980 with a six week history of episodes of giddiness and loss of consciousness. He had recently lost weight. His angina had been treated since early 1977 with perhexiline, $300 \mathrm{mg}$ daily. He had never abused alcohol and his weekly intake averaged less than $20 \mathrm{~g}$. He had taken anticoagulants for several years.

On examination there were signs of mitral stenosis and incompetence, of an old left hemiplegia, and of a sensory peripheral neuropathy. The liver was enlarged $5 \mathrm{~cm}$ below the right costal margin.

Investigations on admission showed: serum bilirubin $17 \mu \mathrm{mol} / \mathrm{l} ; 1 \mathrm{mg} / 100 \mathrm{ml}$ (reference range 5-17 $\mu \mathrm{mol} / 1 ; 0 \cdot 3-1 \mathrm{mg} / 100 \mathrm{ml}$ ), serum aspartate transaminase (AST) $127 \mathrm{U} / \mathrm{l}$ (5-40), serum alkaline phosphatase (ALP) $196 \mathrm{U} / \mathrm{l} ; 28 \mathrm{KAu} / 100 \mathrm{ml}$ (30-110 $\mathrm{U} / \mathrm{l} ; 3-13 \mathrm{KAu} / 100 \mathrm{ml}$ ), plasma albumin $31 \mathrm{~g} / \mathrm{l} ; 3 \cdot 1$ $\mathrm{g} / 100 \mathrm{ml}(35-50 \mathrm{~g} / \mathrm{l} ; 3 \cdot 5-5.0 \mathrm{~g} / 100 \mathrm{ml})$, prothrombin time (PT) (off warfarin), 15s (control 13s), serum HBsAg, smooth muscle (SMA), mitochondrial (AMA) and nuclear (ANF) antibodies negative, serum creatinine $107 \mu \mathrm{mol} / \mathrm{l} ; 1.3 \mathrm{mg} / 100 \mathrm{ml}(60-120$ $\mu \mathrm{mol} / \mathrm{l} ; 0.7-1.4 \mathrm{mg} / 100 \mathrm{ml}$ ). Liver biopsy showed a micronodular cirrhosis with extensive liver cell necrosis, inflammation, and Mallory's hyaline.

Perhexiline was stopped in April 1980, but the patient died in March 1981 following a massive gastrointestinal haemorrhage from oesophageal varices.

DW, a woman aged 72 years, was admitted in April 1981, with a six month history of anorexia, weight loss, proximal muscle weakness and numbness and tingling in her feet. Her angina had been treated for seven months before admission with perhexiline, $200 \mathrm{mg}$ daily. She had never abused alcohol and her weekly intake averaged less than $5 \mathrm{~g}$.

On examination there was severe proximal muscle weakness and a sensory peripheral neuropathy. Her liver was not enlarged.

Investigations on admission showed: bilirubin 10 $\mu \mathrm{mol} / \mathrm{l}(0.6 \mathrm{mg} / 100 \mathrm{ml})$, AST $288 \mathrm{U} / 1$, ALP $76 \mathrm{U} / \mathrm{l}$ $(11 \mathrm{KAu} / 100 \mathrm{ml})$, albumin $34 \mathrm{~g} / \mathrm{l}(3.4 \mathrm{~g} / 100 \mathrm{ml})$, PT 14s, HBsAg, SMA, AMA, ANF negative, creatinine $92 \mu \mathrm{mol} / \mathrm{l}(1.0 \mathrm{mg} / 100 \mathrm{ml})$. Liver biopsy showed a moderately severe hepatitis with a mild degree of fatty infiltration and scanty Mallory's hyaline in the periportal areas.

Perhexiline was withdrawn; her clinical condition improved and five months later her liver function tests were normal apart from an AST value of $54 \mathrm{U} / \mathrm{l}$.

SP, a woman aged 53 years, was admitted in September 1981, with a three month history of anorexia and abdominal distension. Her angina had been treated with perhexiline, $300 \mathrm{mg}$ daily for the previous three years. Acebutolol $200 \mathrm{mg}$ daily controlled her essential hypertension. She had always been teetotal.

On examination her liver was enlarged $7 \mathrm{~cm}$ below the right costal margin and the spleen tip could be felt.

Investigations on admission showed: bilirubin $17 \mu \mathrm{mol} / \mathrm{l}(1 \mathrm{mg} / 100 \mathrm{ml})$, AST $490 \mathrm{U} / \mathrm{l}$, ALP $112 \mathrm{U} / \mathrm{l}$ $(16 \mathrm{KAu} / 100 \mathrm{ml})$, albumin $36 \mathrm{~g} / \mathrm{l}(3.6 \mathrm{~g} / 100 \mathrm{ml})$, PT 17s, HBsAg, SMA, AMA, ANF negative, creatinine $72 \mu \mathrm{mol} / \mathrm{l}(0.8 \mathrm{mg} / 100 \mathrm{ml})$. Liver biopsy showed a micronodular cirrhosis with necrosis, inflammation and numerous Mallory bodies (Fig. 1).

Perhexiline was withdrawn; six months later the patient was clinically improved, the liver function tests showed only minimal abnormalities but the hepatosplenomegaly was unchanged.

AG, a woman aged 69 years, was admitted in January 1982 with a three month history of anorexia, weight loss and abdominal distension. Her angina had been treated for three years with perhexiline, $300 \mathrm{mg}$ daily. She had never abused alcohol and drank minimal quantities at Christmas time only.

On examination she was mildly jaundiced and the liver was enlarged $10 \mathrm{~cm}$ below the right costal margin. There was moderate ascites and a mild sensory peripheral neuropathy.

Investigations on admission showed: bilirubin 21 $\mu \mathrm{mol} / \mathrm{l}(1 \cdot 2 \mathrm{mg} / 100 \mathrm{ml})$, AST $213 \mathrm{U} / \mathrm{l}$, ALP $256 \mathrm{U} / \mathrm{l}$ (36 KAu/100 ml), albumin $29 \mathrm{~g} / \mathrm{l}(2.9 \mathrm{~g} / 100 \mathrm{ml})$, PT 19s, HBsAg, SMA, AMA, ANF negative, creatinine $64 \mu \mathrm{mol} / \mathrm{l}(0.7 \mathrm{mg} / 100 \mathrm{ml})$. Liver biopsy showed a micronodular cirrhosis with massive accumulation of Mallory bodies and associated neutrophils, but only very mild fatty change (Fig. 2).

Perhexiline was withdrawn but the patient developed increasing hepatocellular failure and died one month after admission.

\section{Methods}

PATIENTS

The study group comprised the four patients with perhexiline liver damage. The control group comprised 70 inpatients/outpatients with biopsy proven chronic liver disease. Patients were excluded from the control group if they had alcohol related 
Fig. 1 Liver biopsy from a 53 year old woman who had taken perhexiline, $300 \mathrm{mg}$ daily for three years. Darker nodules containing numerous inflammatory cells are seen in paler connective tissue. Periodic acid - Schiff $\times 75$ (original magnification)

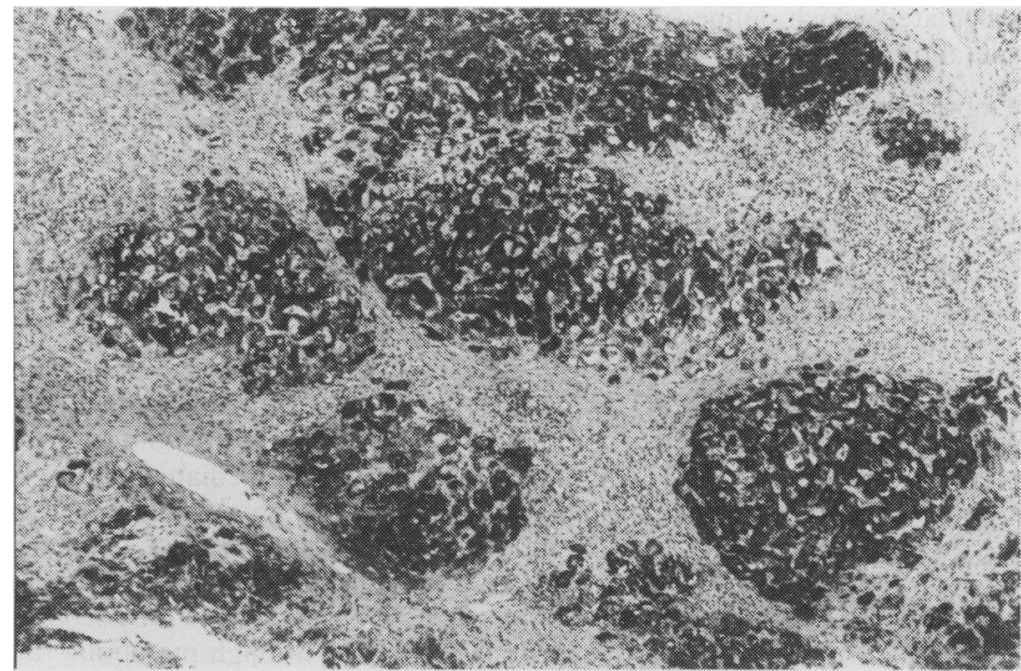

liver disease, were $\mathrm{HBsAg}$ positive, had experienced recent severe gastrointestinal haemorrhage or showed evidence of hepatic encephalopathy or renal failure. Patients with ascites were not studied until their fluid retention had been successfully treated.

In both study and control patients a careful note was made of smoking and drinking habits and of concomitant medication. Prothrombin time, routine liver function tests and plasma creatinine values were measured in all patients using standard laboratory techniques.

All patients were phenotyped for oxidation status using debrisoquine. After an overnight fast a single $10 \mathrm{mg}$ oral dose of debrisoquine (Declinax 10, Roche) was taken. The urine passed in the subsequent eight hours was collected in bulk, the volume measured and a $20 \mathrm{ml}$ sample stored at $-20^{\circ} \mathrm{C}$ until analysis. Each sample was analysed for its content of parent drug and its major metabolite, 4-hydroxydebrisoquine using electron capture gas chromatography. For each patient a metabolic ratio was
Fig. 2 Liver biopsy from a 69 year old woman who had taken perhexiline, $300 \mathrm{mg}$ daily for three years. Swollen hepatocytes contain abundant Mallory bodies (arrowed); there are numerous segmented leucocytes. Haematoxylin and eosin $\times 450$ (original magnification)

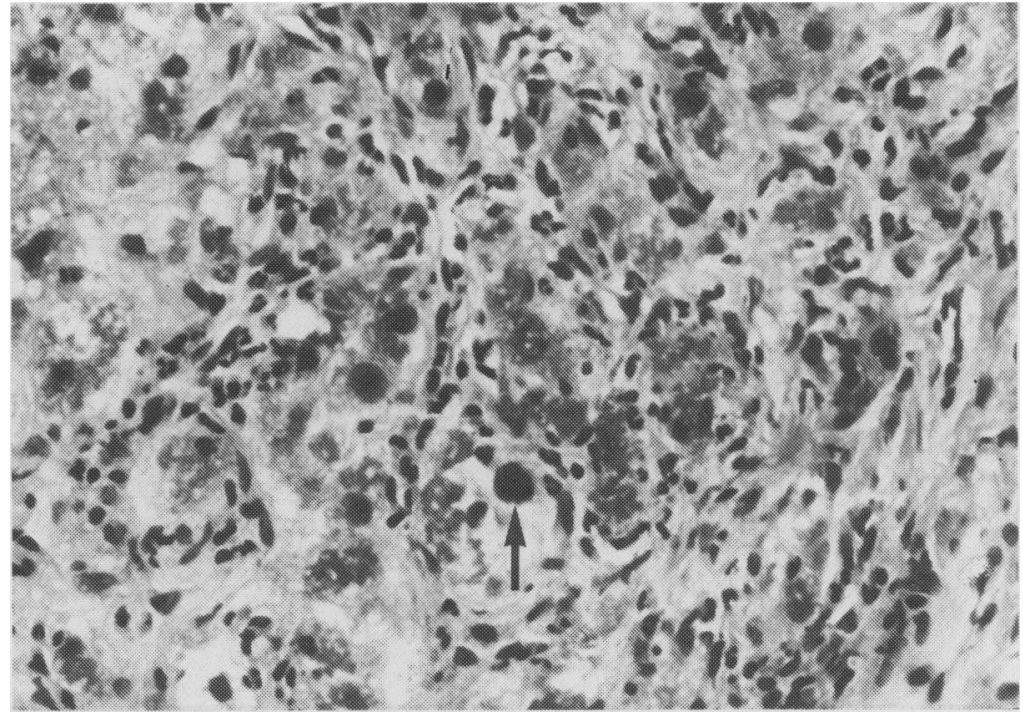


calculated which defined that individual's ability to metabolise the drug:

Debrisoquine metabolic ratio

$$
=\frac{\% \text { excreted as unchanged debrisoquine }}{\% \text { excreted as } 4 \text {-hydroxy-debrisoquine }}
$$

in the $0-8$ hour urine.

Values less than 1 represent extensive oxidation capacity (EM phenotype), while values greater than $12 \cdot 6$ indicate grossly impaired ability as found in individuals homozygous for the $\mathrm{D}^{\mathrm{L}}$ allele (PM phenotype).$^{22}$ Intermediate ratios indicate varying degrees of impairment.

In all subjects studied routine biochemical tests and oxidation phenotyping were performed in the same 24 hour period.

Correlations between variables were performed by linear least squares regression analysis.

\section{Results}

The perhexiline study group comprised three women and one man, mean age 62 years. One female patient smoked, none abused alcohol and most took other medication. The length of treatment and the total dose of perhexiline consumed, the clinical and histological features of toxicity and outcome after drug withdrawal varied (Table 1). In three patients (TB, DW, and SP) debrisoquine phenotyping was undertaken at least eight weeks after perhexiline was withdrawn at which time liver function was improving. In the remaining patient (AG) phenotyping was undertaken one week after drug withdrawal when liver function was deteriorating. All four patients showed metabolic ratios
$>1$; three patients $(75 \%)$ were phenotypically PM with ratios $>12 \cdot 6$ (Table 2 ).

The control group comprised 54 women and 16 men (3F:1M), mean age $49 \cdot 6$ years. Their liver disease varied in aetiology and severity. Thirteen patients had cryptogenic cirrhosis, 27 primary biliary cirrhosis, 24 chronic active hepatitis and six miscellaneous liver diseases; none was severely decompensated. Very few patients smoked and none abused alcohol (Table 3). The majority took prescribed medication including prednisolone (17 patients) and d-penicillamine (10 patients). The metabolic ratios in the control group ranged from $0 \cdot 1$ to $45 \cdot 1$ with a median value of $1 \cdot 2$. Thirty two patients $(45.7 \%)$ had a ratio of $<1$, while six $(8 \cdot 6 \%)$ had ratios $>12 \cdot 6$. The remaining 32 patients $(45.7 \%)$ showed intermediate values (Table 3 ).

The proportion of patients in the study group with high metabolic ratios $(3 / 4)$ was significantly greater than in the control group (6/70) (Fischer's exact probability $F=0.0048)$. Urinary recoveries of debrisoquine and its metabolite were comparable in the perhexiline study group and in controls.

In the control group no correlation existed between the metabolic ratio and the age or sex of the patient or with any particular pattern of cigarette or drug ingestion. Equally no significant relationship existed between the metabolic ratio and values for serum bilirubin $(r=-0 \cdot 014 ; p>0 \cdot 1)$, serum AST $(r=-0.014 ; p>0.1)$, serum ALP $(r=-0.086$; $p>0 \cdot 1)$. plasma albumin $(r=-0.206 ; 0 \cdot 1>p>0 \cdot 05)$ or PT $(r=0.222 ; 0 \cdot 1>p>0.05)$.

\section{Discussion}

Although a dose dependent increase in trans-

Table 1 Clinical and treatment details of four patients with perhexiline liver injury

\begin{tabular}{|c|c|c|c|c|c|c|c|c|c|c|c|}
\hline Patient & $\begin{array}{l}\text { Age } \\
(y r)\end{array}$ & Sex & Alcohol & $\begin{array}{l}\text { Cigarettes } \\
\text { daily }\end{array}$ & $\begin{array}{l}\text { Other } \\
\text { drugs }\end{array}$ & $\begin{array}{l}\text { Length of } \\
\text { treatment } \\
\text { (months) }\end{array}$ & $\begin{array}{l}\text { Total } \\
\text { dose } \\
(g)\end{array}$ & $\begin{array}{l}\text { Weight } \\
\text { loss } \\
(k g)\end{array}$ & $\begin{array}{l}\text { Neuro- } \\
\text { pathy }\end{array}$ & $\begin{array}{l}\text { Liver } \\
\text { lesion }\end{array}$ & $\begin{array}{l}\text { Outcome } \\
\text { after drug } \\
\text { withdrawal }\end{array}$ \\
\hline TB & 54 & $\mathbf{M}$ & - & - & $\begin{array}{l}\text { Warfarin } \\
\text { Spironolactone } \\
\text { Salbutamol }\end{array}$ & 39 & 342 & 6 & + & $\begin{array}{l}\text { Micronodular } \\
\text { cirrhosis } \\
\text { + severe } \\
\text { hepatitis }\end{array}$ & $\begin{array}{l}\text { Died } 11 \\
\text { months } \\
\text { later }\end{array}$ \\
\hline DW & 72 & $\mathbf{F}$ & - & - & Nitrates & 7 & 42 & 12 & + & $\begin{array}{l}\text { Moderate } \\
\text { hepatitis } \\
\text { with fat } \\
+ \text { hyaline }\end{array}$ & Improved \\
\hline SP & 53 & $\mathbf{F}$ & - & 20 & Acebutolol & 36 & 329 & 2 & 0 & $\begin{array}{l}\text { Micronodular } \\
\text { cirrhosis } \\
\text { + severe } \\
\text { hepatitis }\end{array}$ & Improved \\
\hline AG & 69 & $\mathrm{~F}$ & - & - & Nitrates & 36 & 329 & 7 & + & $\begin{array}{l}\text { Micronodular } \\
\text { cirrhosis } \\
+ \text { severe } \\
\text { hepatitis }\end{array}$ & $\begin{array}{l}\text { Died one } \\
\text { month } \\
\text { later }\end{array}$ \\
\hline
\end{tabular}


Table 2 Results of debrisoquine phenotyping in relationship to discontinuation of drug treatment and liver function in four patients with perhexiline liver injury

\begin{tabular}{|c|c|c|c|c|c|c|c|c|}
\hline Patient & $\begin{array}{l}\text { Weeks } \\
\text { between drug } \\
\text { withdrawal } \\
\text { and } \\
\text { phenotyping }\end{array}$ & $\begin{array}{l}\text { Serum } \\
\text { bilirubin } \\
\mu \text { mol/l } \\
(5-17)^{*}\end{array}$ & $\begin{array}{l}\text { Serum } \\
A S T \\
U / l \\
(5-40)\end{array}$ & $\begin{array}{l}\text { Serum } \\
A L P \\
U / l \\
(30-110)\end{array}$ & $\begin{array}{l}\text { Plasma } \\
\text { albumin } \\
\text { g/l } \\
(35-55)\end{array}$ & $\begin{array}{l}P T \\
s \\
\text { (control }=13 \text { ) }\end{array}$ & $\begin{array}{l}\text { Serum } \\
\text { creatinine } \\
\text { 4molll } \\
(60-120)\end{array}$ & $\begin{array}{l}\text { Debrisoquine } \\
\text { metabolic } \\
\text { ratio }\end{array}$ \\
\hline TB & 8 & 10 & 67 & 234 & 24 & $\begin{array}{l}18 \\
\text { (on Warfarin) }\end{array}$ & 107 & $2 \cdot 6$ \\
\hline SP & 24 & 15 & 60 & 150 & 38 & 15 & 70 & $22 \cdot 7$ \\
\hline AG & 1 & 21 & 252 & 337 & 24 & 21 & 64 & $66 \cdot 6$ \\
\hline
\end{tabular}

* Reference range in parenthesis. AST, aspartate transaminase; ALP, alkaline phosphatase; PT, prothrombin time. Bilirubin: $\mu \mathrm{mol} / 1 \times 0.585=\mathrm{mg} / 100 \mathrm{ml}$. ALP: $\mathrm{U} / 1 \times 0.141=\mathrm{KAu} / 100 \mathrm{ml}$. Albumin: $\mathrm{g} / \mathrm{l} \times 0 \cdot 1=\mathrm{g} / 100 \mathrm{ml}$.

Creatinine: $\mu \mathrm{mol} / \mathrm{l} \times 0 \cdot 0113=\mathrm{mg} / 100 \mathrm{ml}$.

aminase values was observed in the first clinical trials with perhexiline, ${ }^{426}$ the demonstration of histological liver injury is more recent. ${ }^{102} 28$ Liver injury ranges from minimal non-specific hepatitis ${ }^{11}$ to micronodular cirrhosis with superimposed features suggesting acute alcoholic hepatitis. $^{1012} 13$ 15-18 2930 The course of the liver disease is variable; usually transaminase values return to normal within a month of perhexiline withdrawal $^{4} 31$ although in some patients the liver lesion progresses despite withdrawal of the drug. ${ }^{11}$ The mortality in patients who develop cirrhosis is high. ${ }^{11-18}$

The incidence of liver injury during prolonged treatment with perhexiline is unknown. Following $600 \mathrm{mg}$ of perhexiline daily for several months, transaminase values invariably rise $; 2$ at a dosage of 200-300 mg per day, the currently recommended therapeutic range, $25-50 \%$ of patients show a transient rise in transaminase values. More sensitive tests, however, reveal a higher degree of abnormalities with $50-75 \%$ of patients showing increased serum bile acid values and increased retention of bromsulphalein (BSP) after three months to two years treatment. ${ }^{3233}$ The incidence of histological liver damage is difficult to assess because few patients are biopsied. Poupon et $a l^{33}$ for example, studied 46 patients on maintenance perhexiline and found hepatomegaly in $13 \%(6 / 46)$, raised serum bile acid values in $57 \%(26 / 46)$ abnormal BSP retention in $74 \%(34 / 46)$ but evidence of histological damage in all 11 patients in whom liver biopsy was undertaken. In the series reported to date 18 patients have shown non-specific hepatitis with or without fatty infiltration, ${ }^{11} 2733-5$ two a granulomatous hepatitis, ${ }^{35} 3617$ an acute or subacute hepatitis closely resembling acute alcoholic hepatitis, ${ }^{811} 14152728$ 37-41 $^{-14}$ and 14 a micronodular cirrhosis with superimposed 'alcoholic' hepatitis. 10-13 $15-18 \quad 2930$

Table 3 Details and results in the control group of patients with chronic liver disease

\begin{tabular}{|c|c|c|c|c|c|c|c|c|c|c|c|}
\hline $\begin{array}{l}\text { Case } \\
\text { no }\end{array}$ & $\begin{array}{l}\text { Age, } \\
\text { sex }\end{array}$ & $\begin{array}{l}\text { Type of } \\
\text { liver } \\
\text { disease* }\end{array}$ & $\begin{array}{l}\text { Cigarettes } \\
\text { daily }\end{array}$ & $\begin{array}{l}\text { Alcohol } \\
\text { consump- } \\
\text { tion }\end{array}$ & $\begin{array}{l}\text { Serum } \\
\text { bilirubin } \\
\mu \text { mol/l } \\
+(5-17)\end{array}$ & $\begin{array}{l}\text { Serum } \\
A S T \\
U / l \\
(5-40)\end{array}$ & $\begin{array}{l}\text { Serum } \\
A L P \\
U / l \\
(30-110)\end{array}$ & $\begin{array}{l}\text { Plasma } \\
\text { albumin } \\
\text { g/l } \\
(35-50)\end{array}$ & $\begin{array}{l}P T \\
s \\
\text { (control } \\
=13)\end{array}$ & 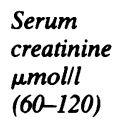 & $\begin{array}{l}\text { Debriso- } \\
\text { quine } \\
\text { metabolic } \\
\text { ratio }\end{array}$ \\
\hline 1 & $52 \mathrm{~F}$ & $\mathrm{CC}$ & - & - & 5 & 46 & 50 & 46 & 14 & 72 & $0 \cdot 1$ \\
\hline 2 & $58 \mathrm{~F}$ & $\mathrm{PBC}$ & - & - & 9 & 57 & 232 & 39 & 12 & 63 & $0 \cdot 1$ \\
\hline 3 & $31 \mathrm{~F}$ & $\mathrm{CAH}$ & - & - & 8 & 28 & 82 & 47 & 13 & 110 & $0 \cdot 2$ \\
\hline 4 & $51 \mathrm{M}$ & PBC & - & - & 18 & 49 & 146 & 36 & 16 & 75 & $0 \cdot 2$ \\
\hline 5 & $33 \mathrm{M}$ & CAH & - & - & 18 & 30 & 90 & 41 & 16 & 71 & $0 \cdot 2$ \\
\hline 6 & $38 \mathrm{M}$ & Haem & - & - & 8 & 28 & 82 & 47 & 13 & 110 & $0 \cdot 2$ \\
\hline 7 & $45 \mathrm{~F}$ & $\mathrm{CC}$ & - & - & 16 & 14 & 112 & 31 & 18 & 73 & $0 \cdot 3$ \\
\hline 8 & $61 \mathrm{~F}$ & PBC & - & - & 18 & 69 & 411 & 40 & 13 & 99 & $0 \cdot 3$ \\
\hline 9 & $64 \mathrm{~F}$ & $\mathrm{CAH}$ & - & - & 6 & 32 & 131 & 46 & 13 & 93 & 0.4 \\
\hline 10 & $65 \mathrm{~F}$ & PBC & - & - & 9 & 35 & 232 & 38 & 13 & 66 & 0.4 \\
\hline 11 & $61 \mathrm{M}$ & Halothane & - & - & 9 & 30 & 63 & 46 & 13 & 95 & 0.4 \\
\hline 12 & $22 \mathrm{M}$ & Wilson's & - & - & 18 & 28 & 72 & 44 & 13 & 79 & 0.5 \\
\hline 13 & $70 \mathrm{M}$ & PBC & - & - & 86 & 163 & 820 & 39 & 12 & 115 & $0 \cdot 5$ \\
\hline
\end{tabular}


Table 3 - continued

\begin{tabular}{|c|c|c|c|c|c|c|c|c|c|c|c|}
\hline $\begin{array}{l}\text { Case } \\
\text { no }\end{array}$ & $\begin{array}{l}\text { Age, } \\
\text { sex }\end{array}$ & $\begin{array}{l}\text { Type of } \\
\text { liver } \\
\text { disease* }\end{array}$ & $\begin{array}{l}\text { Cigarettes } \\
\text { daily }\end{array}$ & $\begin{array}{l}\text { Alcohol } \\
\text { consump- } \\
\text { tion }\end{array}$ & $\begin{array}{l}\text { Serum } \\
\text { bilirubin } \\
\text { pmol/l } \\
+(5-17)\end{array}$ & $\begin{array}{l}\text { Serum } \\
A S T \\
U / I \\
(5-40)\end{array}$ & $\begin{array}{l}\text { Serum } \\
A L P \\
U / l \\
(30-110)\end{array}$ & $\begin{array}{l}\text { Plasma } \\
\text { albumin } \\
\text { g/l } \\
(35-50)\end{array}$ & $\begin{array}{l}P T \\
s \\
\text { (control } \\
=13 \text { ) }\end{array}$ & 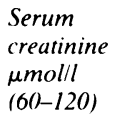 & $\begin{array}{l}\text { Debriso- } \\
\text { quine } \\
\text { metabolic } \\
\text { ratio }\end{array}$ \\
\hline 14 & $53 \mathrm{~F}$ & PBC & 10 & - & 10 & 132 & 534 & 40 & 13 & 102 & 0.5 \\
\hline 15 & $43 \mathrm{~F}$ & CAH & 40 & - & 17 & 52 & 242 & 40 & 13 & 73 & 0.5 \\
\hline 16 & $68 \mathrm{~F}$ & PBC & - & - & 53 & 90 & 1200 & 40 & 13 & 67 & $0 \cdot 6$ \\
\hline 17 & $52 \mathrm{~F}$ & $\mathrm{CAH}$ & - & - & 9 & 92 & 105 & 45 & 13 & 84 & 0.6 \\
\hline 18 & $56 \mathrm{~F}$ & $\mathrm{CAH}$ & - & - & 12 & 42 & 191 & 41 & 14 & 66 & $0 \cdot 7$ \\
\hline 19 & $40 \mathrm{~F}$ & PBC & 20 & - & 90 & 285 & 409 & 40 & 13 & 39 & 0.7 \\
\hline 20 & $50 \mathrm{~F}$ & PBC & - & - & 30 & 45 & 114 & 43 & 13 & 55 & 0.7 \\
\hline 21 & $64 \mathrm{~F}$ & $\mathrm{CAH}$ & - & - & 7 & 20 & 74 & 46 & 14 & 60 & 0.7 \\
\hline 22 & $63 \mathrm{~F}$ & PBC & - & - & 173 & 189 & 1105 & 36 & 13 & 121 & 0.7 \\
\hline 23 & $51 \mathrm{~F}$ & PBC & - & - & 13 & 34 & 325 & 37 & 13 & 55 & 0.8 \\
\hline 24 & $58 \mathrm{~F}$ & PBC & - & - & 18 & 94 & $1200)$ & 40 & 12 & 87 & $0 \cdot 8$ \\
\hline 25 & $64 \mathrm{~F}$ & $\mathrm{CAH}$ & 3 & - & 9 & 353 & 114 & 42 & 15 & 86 & $0 \cdot 8$ \\
\hline 26 & $16 \mathrm{M}$ & Septal & 10 & - & 31 & 40 & 157 & 43 & 14 & 69 & 0.9 \\
\hline 27 & $61 \mathrm{~F}$ & PBC & - & - & 13 & 57 & 350 & 37 & 13 & 50 & 0.9 \\
\hline 28 & $66 \mathrm{~F}$ & PBC & 10 & - & 9 & 30 & 283 & 41 & 12 & 90 & 0.9 \\
\hline 29 & $33 \mathrm{~F}$ & $\mathrm{CAH}$ & - & - & 6 & 57 & 184 & 21 & 16 & 77 & 0.9 \\
\hline 30 & $37 \mathrm{~F}$ & $\mathrm{CC}$ & - & - & 9 & 31 & 76 & 43 & 15 & 65 & $1 \cdot 0$ \\
\hline 31 & $41 \mathrm{~F}$ & $\mathrm{CC}$ & - & - & 14 & 55 & 107 & 36 & 15 & 69 & $1 \cdot 0$ \\
\hline 32 & $46 \mathrm{~F}$ & $\mathrm{CAH}$ & - & - & 12 & 19 & 45 & 40 & 15 & 70 & 1.0 \\
\hline 33 & $60 \mathrm{~F}$ & PBC & - & - & 32 & 142 & 760 & 34 & 17 & 56 & $1 \cdot 1$ \\
\hline 34 & $23 \mathrm{~F}$ & Wilson's & - & - & 16 & 13 & 43 & 46 & 14 & 72 & $1 \cdot 1$ \\
\hline 35 & $57 \mathrm{~F}$ & $\mathrm{CC}$ & - & - & 19 & 32 & 96 & 36 & 16 & 107 & $1 \cdot 1$ \\
\hline 36 & $70 \mathrm{~F}$ & $\mathrm{CAH}$ & - & - & 77 & 33 & 44 & 36 & 14 & 125 & $1 \cdot 2$ \\
\hline 37 & $63 \mathrm{~F}$ & PBC & - & - & 308 & 133 & 518 & 30 & 16 & 86 & $1 \cdot 2$ \\
\hline 38 & $20 \mathrm{~F}$ & Wilson's & - & - & 7 & 29 & 110 & 38 & 15 & 65 & $1 \cdot 2$ \\
\hline 39 & $34 \mathrm{M}$ & $\mathrm{CAH}$ & - & - & 42 & 215 & 192 & 32 & 17 & 79 & $1 \cdot 3$ \\
\hline 40 & $31 \mathrm{M}$ & $\mathrm{CAH}$ & - & - & 14 & 36 & 100 & 43 & 17 & 93 & $1 \cdot 3$ \\
\hline 41 & $67 \mathrm{~F}$ & $\mathrm{CC}$ & - & - & 42 & 28 & 169 & 43 & 14 & 86 & 1.4 \\
\hline 42 & $49 \mathrm{M}$ & $\mathrm{CAH}$ & - & - & 17 & 128 & 100 & 46 & 15 & 77 & $1 \cdot 6$ \\
\hline 43 & $57 \mathrm{~F}$ & $\mathrm{CAH}$ & - & - & 11 & 228 & 72 & 40 & 14 & 79 & 1.7 \\
\hline 44 & $41 \mathrm{M}$ & $\mathrm{CAH}$ & - & - & 25 & 35 & 246 & 28 & 17 & 80 & $2 \cdot 0$ \\
\hline 45 & $63 \mathrm{~F}$ & $\mathrm{PBC}$ & - & - & 11 & 64 & 459 & 41 & 12 & 62 & $2 \cdot 1$ \\
\hline 46 & $43 \mathrm{~F}$ & $\mathrm{CC}$ & - & - & 9 & 55 & 216 & 35 & 14 & 63 & $2 \cdot 1$ \\
\hline 47 & $49 \mathrm{~F}$ & PBC & - & - & 48 & 159 & 592 & 34 & 14 & 72 & $2 \cdot 3$ \\
\hline 48 & $63 \mathrm{~F}$ & $\mathrm{CAH}$ & - & - & 13 & 539 & 272 & 31 & 11 & 57 & $2 \cdot 6$ \\
\hline 49 & $61 \mathrm{~F}$ & $\mathrm{CC}$ & - & - & 5 & 119 & 118 & 42 & 13 & 72 & $2 \cdot 7$ \\
\hline 50 & $57 \mathrm{~F}$ & PBC & - & - & 25 & 55 & 275 & 34 & 15 & 100 & $2 \cdot 7$ \\
\hline 51 & $65 \mathrm{~F}$ & PBC & - & - & 86 & 103 & 935 & 26 & 16 & 71 & $2 \cdot 8$ \\
\hline 52 & $49 \mathrm{~F}$ & $\mathrm{CAH}$ & - & - & 8 & 22 & 325 & 38 & 14 & 105 & $3 \cdot 1$ \\
\hline 53 & $61 \mathrm{~F}$ & PBC & - & - & 309 & 192 & 1765 & 34 & 13 & 84 & $3 \cdot 1$ \\
\hline 54 & $39 \mathrm{~F}$ & PBC & - & - & 58 & 144 & 1020 & 36 & 13 & 63 & $3 \cdot 2$ \\
\hline 55 & $65 \mathrm{~F}$ & $\mathrm{CAH}$ & 10 & - & 49 & 269 & 269 & 25 & 21 & 97 & $3 \cdot 2$ \\
\hline 56 & $51 \mathrm{~F}$ & PBC & - & - & 5 & 32 & 313 & 39 & 12 & 83 & $3 \cdot 7$ \\
\hline 57 & $56 \mathrm{~F}$ & $\mathrm{CAH}$ & - & - & 9 & 24 & 55 & 45 & 13 & 88 & $4 \cdot 6$ \\
\hline 58 & $60 \mathrm{~F}$ & PBC & - & - & 13 & 89 & 446 & 42 & 12 & 72 & $5 \cdot 1$ \\
\hline 59 & $34 \mathrm{~F}$ & PBC & - & - & 74 & 93 & 326 & 33 & 15 & 64 & $5 \cdot 3$ \\
\hline 60 & $45 \mathrm{M}$ & $\mathrm{CC}$ & - & - & 9 & 89 & 88 & 24 & 21 & 82 & $6 \cdot 2$ \\
\hline 61 & $31 \mathrm{~F}$ & $\mathrm{CC}$ & - & - & 37 & 126 & 256 & 23 & 21 & 57 & $6 \cdot 8$ \\
\hline 62 & $27 \mathrm{~F}$ & $\mathrm{CAH}$ & - & - & 68 & 196 & 267 & 20 & 22 & 109 & $7 \cdot 8$ \\
\hline 63 & $44 \mathrm{~F}$ & $\mathrm{CC}$ & - & - & 51 & 68 & 241 & 27 & 18 & 61 & $8 \cdot 4$ \\
\hline 64 & $28 \mathrm{~F}$ & $\mathrm{CAH}$ & - & - & 26 & 48 & 103 & 36 & 18 & 65 & $8 \cdot 8$ \\
\hline 65 & $63 \mathrm{M}$ & PBC & - & - & 36 & 118 & 612 & 31 & 15 & 101 & $13 \cdot 7$ \\
\hline 66 & $54 \mathrm{M}$ & $\mathrm{CC}$ & - & - & 27 & 32 & 77 & 39 & 18 & 88 & $15 \cdot 6$ \\
\hline 67 & $54 \mathrm{~F}$ & PBC & - & - & 5 & 42 & 497 & 39 & 12 & 67 & $20 \cdot 7$ \\
\hline 68 & $31 \mathrm{~F}$ & $\mathrm{CAH}$ & - & - & 22 & 208 & 125 & 31 & 21 & 71 & $21 \cdot 5$ \\
\hline 69 & $50 \mathrm{M}$ & $\mathrm{CAH}$ & - & - & 11 & 52 & 86 & 39 & 15 & 74 & $35 \cdot 6$ \\
\hline 70 & $41 \mathrm{M}$ & CC & - & - & 46 & 44 & 91 & 34 & 16 & 92 & $45 \cdot 1$ \\
\hline
\end{tabular}

Halothane, cirrhosis following halothane hepatitis: Septal incomplete septal fibrosis.

+ reference range in parenthesis; AST, aspartate transaminase; ALP, alkaline phosphatase; PT, prothrombin time.

Bilirubin: $\mu \mathrm{mol} / / \times 0 \cdot 585=\mathrm{mg} / 100 \mathrm{ml}$. ALP: $\mathrm{U} / 1 \times 0 \cdot 141=\mathrm{KAu} / 100 \mathrm{ml}$. Albumin: $\mathrm{g} / \mathrm{l} \times 0 \cdot 1=\mathrm{g} / 100 \mathrm{ml}$.

Creatinine: $\mu \mathrm{mol} / \mathrm{l} \times 0.0113=\mathrm{mg} / 100 \mathrm{ml}$ 
The liver damage in the present four patients almost certainly resulted from perhexiline. None of the patients consumed excessive quantities of alcohol nor took other, potentially hepatotoxic drugs. Other manifestations of perhexiline toxicity were present; two patients showed disturbed balance (TB, DW), three showed significant weight loss (TB, DW, AG), three had peripheral neuropathy (TB, DW, AG) and one a proximal myopathy (DW). The liver lesions were similar to those already described following perhexiline and no other cause for the liver disease was found. Withdrawal of the drug resulted in a prompt improvement in the clinical condition and some biochemical tests in two patients; the remaining two patients deteriorated despite drug withdrawal, a course already well recognised after perhexiline liver injury. ${ }^{12} 13151718 \quad 30$

Severe hepatotoxicity only occurs in a percentage of perhexiline treated patients. In the present study all four patients with perhexiline liver injury showed diminished drug elimination ability as measured by debrisoquine hydroxylation. In three of the four patients the metabolic ratio exceeded 12.6 indicating the PM phenotype. In the control group of 70 patients with chronic liver disease the proportion of patients with the PM phenotype was similar to that in the healthy population ${ }^{22}$ and there was no correlation between oxidation capacity and standard liver function tests. Thus the impairment of drug oxidation ability in the perhexiline damaged patients can not be explained simply on the basis of impaired hepatocellular function.

A clear association therefore exists between perhexiline liver injury and diminished drug oxidation capacity. As a result of impaired drug oxidation, perhexiline would be expected to accumulate $^{19}$ and this might explain the toxic sequelae. The actual mechanism of the toxic reaction is unknown, but drug induced disturbances of phospholipid metabolism have been implicated. ${ }^{12}$

One of the four patients studied had a metabolic ratio of 2.6 which indicates a substantial metabolic impairment, but not the PM phenotype. Thus although drug oxidation status may be a major determinant of both therapeutic and toxic drug responses, other factors must also be important; HLA-B8 might be one such factor. ${ }^{42}$

Perhexiline maleate is a useful agent in the management of intractable angina. Nevertheless the severe toxic side effects have limited its use. While routine assessment of oxidation status would allow more selective use of the drug this facility is not generally available. Thus careful monitoring of liver function is required in all patients prescribed perhexiline and prompt withdrawal indicated if liver function tests become persistently abnormal on therapeutic doses.

We would like to thank the physicians who referred patients for study: Drs W D Alexander, R S Elkeles, Professor R Fox and Dr R R Ghose. Professor P J Scheuer kindly reassessed the liver biopsies and provided the figures. MYM is William Gibson Scholar of the Royal Society of Medicine. RR was supported by the Lewis Fellowship Fund. RRS and NSO were supported by the Wellcome Trust. Merrell Pharmaceuticals Ltd provided financial support.

\section{References}

1 Armstrong ML. A comparative study of perhexiline, beta-adrenergic blocking agents and placebos in the management of angina pectoris. Postgrad Med J 1973; 49: suppl 3: 108-11.

2 Dettori AG, Malagnino G, Fatt F, Oriani G. Perhexiline versus prenylamine. A controlled clinical trial in coronary insufficiency. Postgrad Med J 1973; 49: suppl 3: 113-4.

3 Cawein MK, Lewis RE, Hudak WJ, Hoekenga MT. Clinical evaluation of perhexiline maleate in patients with angina pectoris associated with a positive coronary artery disease index. Postgrad Med J 1973; 49: suppl 3: 121-4.

4 Pilcher J, Chandrasekhar KP, Russell RJ, Boyce MJ, Pierce TH, Ikram H. Long-term assessment of perhexiline maleate in angina pectoris. Postgrad Med J 1973; 49: suppl 3: 115-8.

5 Gitlin N, Nellen M. Perhexiline maleate in the treatment of angina pectoris: a double-blind trial. Postgrad Med J 1973; 49: suppl 3: 100-4.

6 Houdent CE, Wolf LM, Corriat A. Liver during perhexiline hypoglycaemia. Lancet 1977; 2: 1028.

7 Tomlinson IW, Rosenthal FD. Proximal myopathy after perhexiline maleate treatment. $\mathrm{Br}$ Med J 1977; 1: $1319-20$

8 Abaza A, Cattan D, Aziza C, Pappo E. Effects de secondaires mais réversibles à la prise de perhexiline. Nouv Presse Méd 1973; 2: 2820.

9 Laplane D, Bousser MG, Bouche P, Touboul PJ. Peripheral neuropathies caused by perhexiline maleate. In: International symposium on perhexiline maleate. Amsterdam: Excerpta Medica (Congress Series 424), 1978: 89-96.

10 Pelletier M. Lambert R, Paliard P, Bory R. Hépatite toxique subaigue après traitement par maléate de perhexiline. Nouv Presse Méd 1976; 5: 1070.

11 Beaugrand M, Poupon R, Lévy VG, et al. Lésions hépatiques dues au maléate de perhexiline. Gastroenterol Clin Biol 1978; 2: 579-88. 
12 Pessayre D, Bichara M, Feldman G, Degott C, Potet F, Benhamou J-P. Perhexiline maleate-induced cirrhosis. Gastroenterology 1979; 76: 170-7.

13 Bonnet P, Jourdan J, Janbon F, Michel H, Bertrand A. Cirrhose hépatique après deux ans de traitement par le maléate de perhexiline. Nouv Presse Méd 1978; 7: 208.

14 Lenoir C, Blanchon P. Hépatites due au maléate de perhexiline. Coeur Méd Interne 1978; 17: 69-75.

15 Paliard P, Vitrey D, Fournier G, Belhadjali J, Patricot L, Berger F. Perhexiline maleate-induced hepatitis. Digestion 1978; 17: 419-27.

16 Forbes GB, Rake MO, Taylor DJE. Liver damage due to perhexiline maleate. J Clin Pathol 1979; 32: 1282-5.

17 Paccalin J, Rumeau J-M, Lagueny A, et al. Analyses des effects secondaries du maléate de perhexiline. Bordeaux Med 1979; 12: 789-93.

18 Roche J, Fournet J, Meullener J, Faure H, Massot C. Insuffisance hépatique aigue due au maléate de perhexiline. Nouv Presse Méd 1979; 8: 521-2.

19 Wright GJ, Leeson GA, Zeiger AV, Lang JW. The absorption, excretion and metabolism of perhexiline maleate by the human. Postgrad Med J 1973; 49: suppl 3: 8-15.

20 Mahgoub A, Idle JR, Dring LG, Lancaster R, Smith RL. Polymorphic hydroxylation of debrisoquine in man. Lancet 1977; 2: 584-6.

21 Sloan TP, Mahgoub A, Lancaster R, Idle JR, Smith RL. Polymorphism of carbon oxidation of drugs and clinical implications. Br Med J 1978; 2: 655-7.

22 Price-Evans DA, Mahgoub A, Sloan TP, Idle JR, Smith RL. A family and population study of the genetic polymorphism of debrisoquine oxidation in a British white population. J Med Genet 1980; 17: 102-5.

23 Shah RR, Oates NS, Idle JR, Smith RL, Lockhart JDF. Impaired oxidation of debrisoquine in patients with perhexiline neuropathy. $\mathrm{Br}$ Med $J$ 1982; 284: 295-9.

24 Kahn GC, Boobis AR, Murray S, Brodie MJ, Davies DS. Assay and characteristics of debrisoquine 4hydroxylase activity of microsomal fractions of human liver. Br J Clin Pharmacol 1982; 13: 637-45.

25 Lieber CS, DeCarli LM. The role of hepatic microsomal ethanol oxidizing systems (MEOS) for ethanol metabolism in vivo. J Pharmacol Exp Ther 1972; 181: 279-87.

26 Newberne JW. Assessment of safety data from patients on short- and long-term perhexiline therapy. Postgrad Med J 1973; 49: suppl 3: 125-9.

27 Beaugrand $M$. Hépatite medicamenteuse à la perhexiline. Nouv Presse Méd 1976; 5: 1695.

28 Herne N, Raillat A, Marion J, Le Gall F, Chagnon A. Hépatite toxique sub-aigue et maléate de perhexiline. Nouv Presse Méd 1976; 5: 2164.

29 Hauw J-J, Singer B, Poupon R, et al. Inclusions polymorphes diffuses chez un malade traité par le maléate de perhexiline. Nouv Presse Méd 1978; 7: 817-20.

30 Beaugrand $\mathbf{M}$, Chousterman M, Callard P, Camilleri J-P, Petite J-P, Ferrier J-P. Hépatites au maléate de perhexiline (Pexid ${ }^{\circledR}$ ) évoluant ver la cirrhose malgré l'arrêt du traitment (2 cas). Gastroenterol Clin Biol 1977; 1: 745-50.

31 Claudel S, Lery N, Boissel J-P, Paliard P. Foie et maléate de perhexiline (Pexid ${ }^{\circledR}$ ). À propos de 100 observations. Ann Gastroentérol Hépatol 1979; 15: 341-6.

32 Howard DJ, Russell Rees J. Long-term perhexiline maleate and liver function. Br Med J 1976; 1: 133.

33 Poupon R, Rosensztajin L, de Saint-Maur P-P, Lageron A, Gombeau T, Darnis F. Perhexiline maleate-associated hepatic injury prevalence and characteristics. Digestion 1980; 20: 145-50.

34 Kopelman P, Morgan PGM. Liver damage after perhexiline maleate. Lancet 1977; 1: 705.

35 Long JP, Fitzgerald O, Maurer BJ. Hepatotoxicity following treatment with perhexiline maleate. J Ir Med Assoc 1980; 73: 275-6.

36 Stentiford NH. Perhexiline maleate: a case of severe jaundice. Br J Clin Pract 1977; 31: 136-7.

37 Blanchon P, Paillas J, Lenoir C, Vieillefond A, Lauriat $H$. Documents anatomiques concernant un accident mortel dû à un traitment prolongé par le maléate de perhexiline. Ann Med Interne (Paris) 1977; 128: 715-8.

38 Lubetzki J, Warnet A, Kaloustian E, Fusciardi J. Complications multiples d'un traitment par le maléate de perhexiline. Ann Med Interne (Paris) 1977; 128: 719-22.

39 McDonald GS. Liver damage after perhexiline maleate. Lancet 1977; 1: 705.

40 Serot J-M, Floquet A, Penin F, Cuny G, Griguon G. Ultrastructural study of the liver following treatment with perhexiline maleate. Sem Hôp Paris 1977; 53: 2199-200.

41 Lewis D, Wainwright HC, Kew MC, Zwi S, Isaacso C. Liver damage associated with perhexiline maleate. Gut 1979; 20: 186-9.

42 Davies P, Moulder C. Perhexiline hepatitis and HLA B8. Lancet 1982; 2: 109. 